OPEN ACCESS

Edited by:

Behnam Rezai Jahromi, Helsinki University Central Hospital,

Finland

Reviewed by:

Edgar A. Samaniego,

The University of lowa, United States

Antti Lindgren,

Kuopio University Hospital, Finland

Seyedeh Fatemeh Salimi Ashkezari,

George Mason University,

United States

*Correspondence:

Sylvia Saalfeld

sylvia.saalfeld@ovgu.de

Specialty section:

This article was submitted to

Endovascular and Interventional

Neurology,

a section of the journal

Frontiers in Neurology

Received: 06 September 2021

Accepted: 08 December 2021

Published: 24 January 2022

Citation:

Saalfeld S, Stahl J, Korte J, Miller

Marsh LM, Preim B, Beuing $O$,

Cherednychenko $Y$, Behme $D$ and

Berg P (2022) Can Endovascular

Treatment of Fusiform Intracranial

Aneurysms Restore the Healthy

Hemodynamic Environment?-A Virtual

Pilot Study. Front. Neurol. 12:771694.

doi: 10.3389/fneur.2021.771694

\section{Can Endovascular Treatment of Fusiform Intracranial Aneurysms Restore the Healthy Hemodynamic Environment?-A Virtual Pilot Study}

\author{
Sylvia Saalfeld ${ }^{1,2 *}$, Janneck Stahl ${ }^{1,3}$, Jana Korte ${ }^{1,2}$, Laurel Morgan Miller Marsh ${ }^{1,3,4}$, \\ Bernhard Preim ${ }^{2}$, Oliver Beuing ${ }^{5}$, Yurii Cherednychenko ${ }^{6}$, Daniel Behme ${ }^{1,7}$ and \\ Philipp Berg ${ }^{1,3}$
}

\begin{abstract}
'Research Campus STIMULATE, University of Magdeburg, Magdeburg, Germany, ${ }^{2}$ Department of Simulation and Graphics, University of Magdeburg, Magdeburg, Germany, ${ }^{3}$ Department of Fluid Dynamics and Technical Flows, University of Magdeburg, Magdeburg, Germany, ${ }^{4}$ Department of Mechanical Engineering, University of Washington, Seattle, WA, United States, ${ }^{5}$ Department of Radiology, AMEOS Hospital Bernburg, Bernburg, Germany, ${ }^{6}$ Endovascular Centre, Dnipropetrovsk Regional Clinical Hospital named after l.I. Mechnikov, Dnipro, Ukraine, ${ }^{7}$ Department of Neuroradiology, University Hospital Magdeburg, Magdeburg, Germany
\end{abstract}

Numerous studies assess intracranial aneurysm rupture risk based on morphological and hemodynamic parameter analysis in addition to clinical information such as aneurysm localization, age, and sex. However, intracranial aneurysms mostly occur with a saccular shape located either lateral to the parent artery or at a bifurcation. In contrast, fusiform intracranial aneurysms (FIAs), i.e., aneurysms with a non-saccular, dilated form, occur in approximately $3-13 \%$ of all cases and therefore have not yet been as thoroughly studied. To improve the understanding of FIA hemodynamics, this pilot study contains morphological analyses and image-based blood flow simulations in three patient-specific cases. For a precise and realistic comparison to the pre-pathological state, each dilation was manually removed and the time-dependent blood flow simulations were repeated. Additionally, a validated fast virtual stenting approach was applied to evaluate the effect of virtual endovascular flow-diverter deployment focusing on relevant hemodynamic quantities. For two of the three patients, post-interventional information was available and included in the analysis. The results of this numerical pilot study indicate that complex flow structures, i.e., helical flow phenomena and the presence of high oscillating flow features, predominantly occur in FIAs with morphologically differing appearances. Due to the investigation of the individual healthy states, the original flow environment could be restored which serves as a reference for the virtual treatment target. It was shown that the realistic deployment led to a considerable stabilization of the individual hemodynamics in all cases. Furthermore, a quantification of the stent-induced therapy effect became feasible for the treating physician. The results of the morphological and hemodynamic analyses in this pilot study show that virtual stenting can be used in FIAs to quantify the effect of the planned endovascular treatment.

Keywords: fusiform intracranial aneurysm, hemodynamic simulation, virtual stent deployment, blood flow patterns, endovascular treatment 


\section{INTRODUCTION}

Fusiform intracranial aneurysms (FIAs) are circumferential dilatations of the cerebral vessels leading to pathological hemodynamics. As a consequence, the vessel wall can be drastically weakened followed by a potential rupture with a subsequent subarachnoid hemorrhage. Compared to saccular intracranial aneurysms, FIAs have a lower prevalence in the Western population, where two large autopsy series indicate a prevalence of only $<0.1 \%(1-3)$. More recently, this value is estimated to be somewhere between 3 and 13\% (4). However, FIAs more often occur at the posterior cerebral circulation, can strongly vary in size (diameter and length), and are more often present in young patients with a higher prevalence in men when compared to saccular IAs which have a 3-fold larger prevalence in women (5).

Due to their rarity, FIAs have not been as well studied as saccular aneurysms including morphological and hemodynamic parameter analysis with respect to rupture risk. For instance, morphological parameter analysis usually requires the presence of an aneurysm neck in order to extract features like maximal height or aspect ratio (6). Furthermore, the individual hemodynamic state is difficult to assess, since a clear distinction between the aneurysmal lumen and the parent vessel can be highly complicated.

Regarding the treatment of FIAs, an increasing number of minimally-invasive techniques exists (7). A multicenter study found that FIAs possess the lowest occlusion rate and were associated with most complications during the interventions (8). Furthermore, sufficient device selections can be complicated and undesired treatment effects such as intimal hyperplasia have been shown to be related to insufficient flow-diverter placement $(9,10)$.

This pilot study focuses on the morphological and hemodynamic analysis of these rare pathologies by comparing three representative FIAs. For assessing the potentially pathologic hemodynamics leading to the formation of the aneurysms, all FIAs were manually removed for healthy counterparts. Furthermore, realistic treatments using virtual flow-diverter deployments were carried out to quantify the efficacy of each patient-specific treatment scenario. This helps to answer the question of whether endovascular treatment of FIAs restores the healthy pre-pathological hemodynamic state.

\section{MATERIALS AND METHODS}

This section describes the datasets of our pilot study and explains the subsequent analysis steps.

\subsection{Patient Data}

Due to the absence of high-quality image data, only three patientspecific fusiform aneurysms were analyzed in this study (refer to Figure 1). The selection was based on Huber's definition, where the lesions present with an arterial dilation larger than $1.5 \times$ of the nominal vessel diameter without possessing a neck. Since FIAs are not as common as their saccular counterparts, there was only a limited number available.
For Case 1, a 63-year-old male patient underwent computer tomographic angiography with a resolution of $0.47 \times 0.47$ $\times 0.5 \mathrm{~mm}^{3}$. The patient suffered from an FIA at the left vertebral artery (Refer to Figure 1). Furthermore, he presented with a subarachnoid hemorrhage which required immediate treatment. Although the stenting was considered successful, the patient suffered from generally poor health and no long-term information was available. For Case 2, a 34-year-old female patient with a FIA at the left middle cerebral artery underwent 3D digital subtraction imaging (3D DSA) with a resolution of $0.28 \times 0.28 \times 0.28 \mathrm{~mm}^{3}$. The patient stopped treatment and did not appear for follow-up visits. Case 3 was acquired as $3 \mathrm{D}$ DSA data $\left(0.36 \times 0.36 \times 0.36 \mathrm{~mm}^{3}\right)$ from a 48 years old man with a basilar fusiform aneurysm. A Leo stent $(5.5 \times 75)$ was implanted, yielding the desired neurological improvement and aneurysm elimination.

\subsection{Segmentation and Extraction of the Healthy Counterparts}

For each of the patient-specific fusiform data sets, a thresholdbased segmentation was applied to yield 3D surface models based on prior work (11). During segmentation, we have been focusing on a large vascular domain, i.e., keeping as much of the peripheral arteries as possible depending on the acquired image data (refer to Figure 1). For subsequent post-processing steps, the vessel centerline was extracted with the Vascular Modeling Toolkit (VMTK) (12). In order to restore the healthy variants of the three FIA models and to compare them with the original shapes, manual Laplacian smoothing was applied locally using the open source 3D creation suite Blender 2.9 (Blender Foundations, Amsterdam, Netherlands). For this purpose, the sculpt mode can be used in Blender 2.9. A circular region of interest is available in which Laplacian smoothing is performed on the corresponding section of the surface. With this tool, the pathological part of the vessel was smoothed and shrunk based on the proximal and distal vessel diameter of the healthy parent vessel. This step was conducted by a single medical engineer.

\subsection{Fast Virtual Stenting}

To allow for an investigation of the efficacy of endovascular treatment, a fast virtual stenting (FVS) approach was applied to all three FIA cases. A flow-diverting device was virtually deployed using in-house software, which allows for the replication of arbitrary stent properties within minutes (13). The consideration of the discrete stent struts as well as the vessel-induced stent shortening or elongation allows for realistic reproduction of a possible treatment scenario as well as the consideration of flow phenomena occurring in the vicinity of the flow-diverters. The deployment results are displayed in Figure 2, and the corresponding properties are summarized in Table 1. For further details, the interested reader is referred to Berg et al. (14).

\subsection{Blood Flow Simulation}

Image-based blood flow simulations were performed via computational fluid dynamics. Spatial discretization of the models resulted in the generation of fine, polyhedral meshes with a base size of $0.15 \mathrm{~mm}$ in accordance with recent 


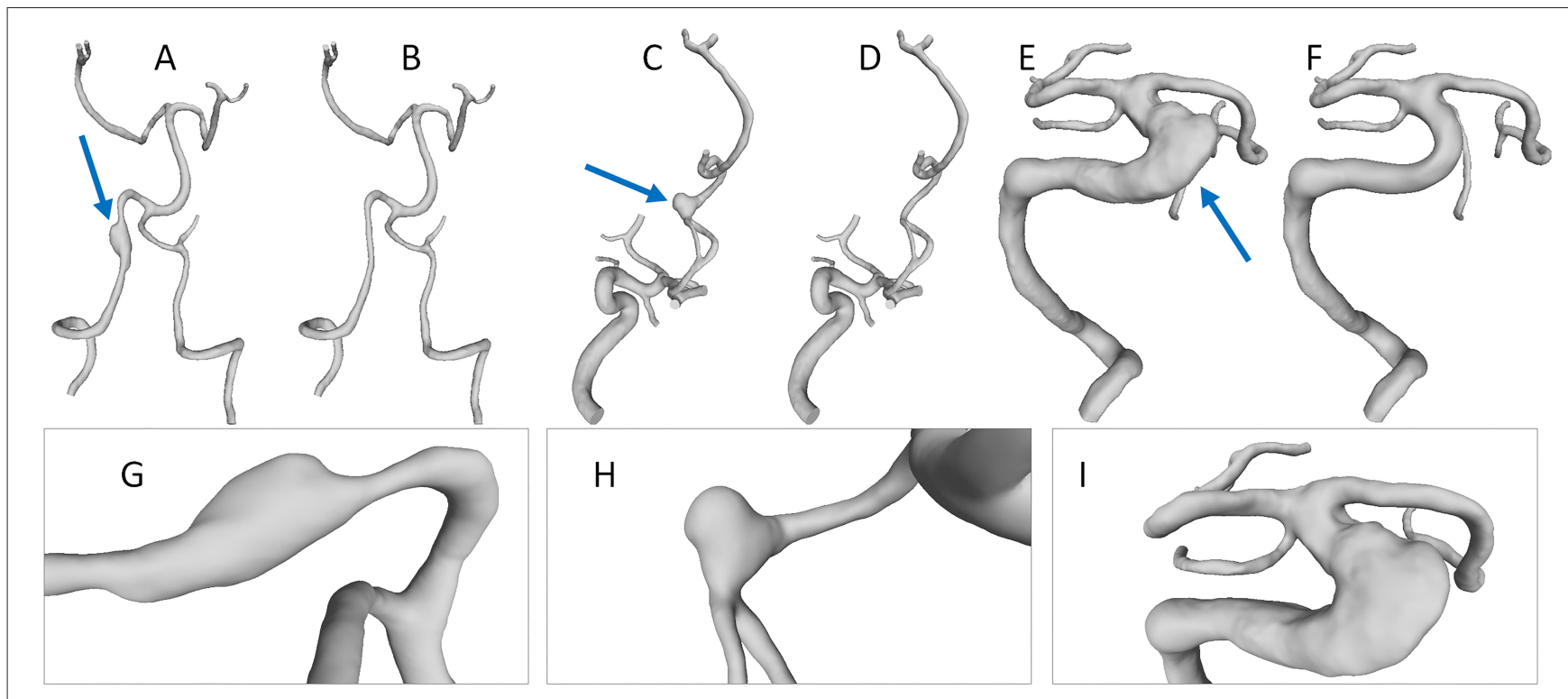

FIGURE 1 | Depiction of the three fusiform aneurysm cases (marked with an arrow) and their corresponding healthy 3D models. Case 1 is depicted in (A,B). Case 2 is depicted in (C, $\mathbf{D})$ and Case 3 is depicted in $\mathbf{( E , F ) , ~ r e s p e c t i v e l y . ~ F u r t h e r m o r e , ~ f o r ~ e a c h ~ f u s i f o r m ~ a n e u r y s m ~ c a s e , ~ a ~ d e t a i l e d ~ p r e s e n t a t i o n ~ i s ~ g i v e n ~ i n ~} \mathbf{( G - I ) . ~}$

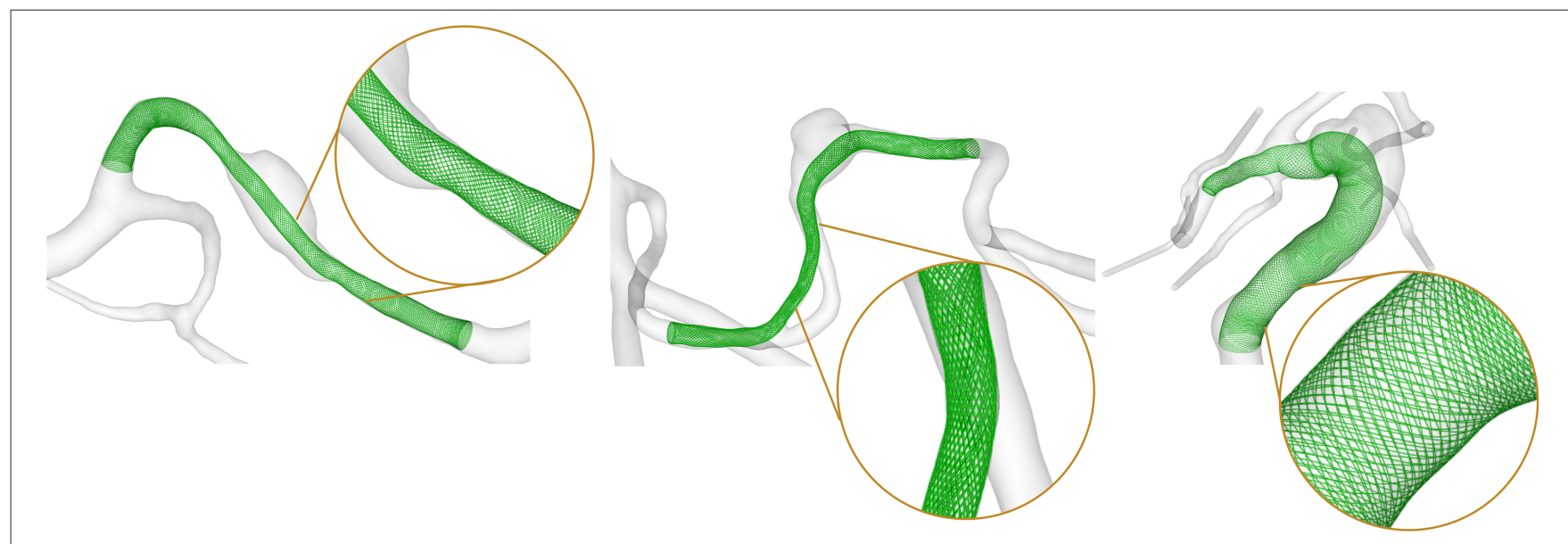

FIGURE 2 | Results of the virtual flow-diverter deployment for Case 1 (left), Case 2 (center), and Case 3 (right). Inlays show the explicit resolution of the braided stent structure allowing for a precise evaluation of the post-interventional hemodynamic state.

TABLE 1 | Nominal geometric parameters of the virtual flow-diverter stents for each patient-specific FIA.

\begin{tabular}{lcccc}
\hline Case & $\begin{array}{c}\text { Length } \\
{[\mathrm{mm}]}\end{array}$ & $\begin{array}{c}\text { Diameter } \\
{[\mathrm{mm}]}\end{array}$ & $\begin{array}{c}\text { Number } \\
\text { of wires }\end{array}$ & $\begin{array}{c}\text { Strut thickness } \\
{[\mu \mathrm{m}]}\end{array}$ \\
\hline 1 & 20 & 3 & 48 & 42 \\
2 & 10 & 2.25 & 48 & 42 \\
3 & 60 & 7 & 52 & 42 \\
\hline
\end{tabular}

recommendations in prior study (15). This yielded a total number of elements ranging from 0.8 to 8.9 million, depending on the considered vessel section and configuration. High element numbers are caused by the precise description of individual stent struts.

Due to the absence of patient-specific flow measurements, boundary conditions for in and outflow cross-sections were employed. At each inlet of the considered cases, representative time-dependent flow rates from precise $4 \mathrm{D}$ phase-contrast MRI measurement conducted in a healthy volunteer were scaled according with the individual inlets of Cases 1-3 (16). Since the segmented vascular domains contain a high number of outlets, we used an advanced flow-splitting technique for realistic splitting ratios based on the underlying vessel shape (17) instead of the commonly used zero-pressure or generalized approaches based on Murray's. Further assumptions for the time-dependent simulations include the treatment of blood as an incompressible 
$\left(\rho=1,055 \frac{\mathrm{kg}}{\mathrm{m}^{3}}\right)$, Newtonian $(v=4 \mathrm{mPa} \cdot \mathrm{s})$ fluid, and the consideration of laminar flow behavior. For each of the nine simulations, two cardiac cycles were simulated, while the first one was discarded, and the last one was included in the advanced analysis.

\subsection{Analysis}

For the three fusiform aneurysms, qualitative and the quantitative analysis was carried out. Since morphological parameters mostly require a saccular shape, we focused on the vessel cross-section. In order to specifically consider elliptical vessel cross-sections, we utilized previous study to extract the vessel cross-sectional area along the vessel's centerline (17). In this study, rays are cast perpendicular to the vessel's centerline to determine the cross-section of the area.

Next, relevant hemodynamic parameters that were associated with an increased rupture risk in previous studies were extracted. The focus is set on shear-stress and flow-related effects (18-20):

- Time-averaged Wall Shear Stress (AWSS)

- Oscillatory Shear Index (OSI)

- Relative Residence Time (RRT)

- Oscillatory Velocity Index (OVI)

- Kinetic Energy (KE)

A detailed description of these parameters is provided in the Supplementary Material.

\section{RESULTS}

For each FIA case, the maximum cross-sectional area is evaluated. This is followed by an analysis of the hemodynamic parameters, including a comparison with the manually-created healthy counterparts, and the post-interventional states based on the virtual deployment.

\subsection{Case 1}

For morphological evaluation, we extracted the FIA's crosssectional areas perpendicular to the vessel's centerline, which are listed in Table 2. The FIA of Case 1, located on the patient's vertebral artery, had a maximum cross-sectional area of approximately $14.53 \mathrm{~mm}^{2}$, which is a relative increase in comparison to the parent vessel's of $345 \%$.

Selected results of the time-dependent blood flow simulations are presented in Figure 3. The blood flow pattern for the FIA shows a small vortex in the aneurysmal lumen indicating a complex flow structure (refer to inlay). When analyzing the healthy model, no vortex or disturbance of the blood flow is perceivable. The simulation of the virtual stenting configuration yields a hemodynamic state, where no vortex remains (refer to inlay). When analyzing the AWSS, an area with elevated AWSS values is apparent proximal to the aneurysm (refer to arrowheads, Figure 3), which drastically decreases in the aneurysmal lumen. Distal to the aneurysm, an area with higher AWSS values remains, independent of the healthy or virtually treated versions (refer to arrows, Figure 3). Regarding OSI, the post-interventional configuration yields a reduction of this parameter as well (refer to green circles, Figure 3), which is
TABLE 2 | Morphological analysis for each case, where max.

\begin{tabular}{|c|c|c|c|}
\hline Case & $\operatorname{Max} . C S A_{A}\left(\mathrm{~mm}^{2}\right)$ & $C S A_{P V}\left(\mathrm{~mm}^{2}\right)$ & $R I(\%)$ \\
\hline 1 & 14.53 & 4.21 & 345 \\
\hline 2 & 7.40 & 1.24 & 581 \\
\hline 3 & 70.51 & 22.61 & 312 \\
\hline
\end{tabular}

$C A S_{A}$ denotes the maximum cross-sectional area (perpendicular to the centerline) of the fusiform aneurysm and CSA $A_{P V}$ denotes the cross-sectional area (perpendicular to the centerline) of the parent vessel proximal to the aneurysm. Rl denotes the relative increase as a percentage, i.e., (max. $\left.C S A_{A} / C S A_{P V} \cdot 100 \%\right)$.

desired as visible in the healthy version, where no elevated OSI is perceived. This is also reflected by the $R R T$ analysis. In this study, virtual stenting yields a more homogeneous distribution of blood flow with respect to $R R T$ when compared to the untreated dataset (refer to asterix in Figure 3).

Besides the shear-related quantities, the analysis of OVI and $K E$ enables an assessment of the time-dependent dynamics within the vascular lumen. Specifically, regions of increased oscillatory velocity are present in the FIA Case 1, which vanish in the corresponding healthy vessel section (refer to Figure 3). After virtually deploying a flow-diverting device, these regions can be reduced toward a healthy state. However, a small region of increased flow disturbance remains in the distal part of the aneurysm, which is also detectable by $K E$.

\subsection{Case 2}

The FIA comprises two inlets, a challenge for the treating physician. Although it is smaller than the other cases, it exhibits the largest increase of diameter compared to the parent vessel, recall Table 2. Hence, the maximum cross-sectional area of the FIA is approximately $7.40 \mathrm{~mm}^{2}$, which is $581 \%$ of the parent vessel's cross-sectional area of $1.24 \mathrm{~mm}^{2}$. Compared to Case 1 , the FIA presents with a more spherical shape, and due to the relatively small volume, no complex flow patterns develop within the sac.

However, when analyzing the shear-related parameters, an area of slightly elevated AWSS values is apparent in the manuallycreated healthy version and in the virtually-treated one (refer to arrowheads in Figure 4). In the FIA model, this area has considerably lower AWSS values. Furthermore, OSI analysis reveals a clear reduction of this hemodynamically relevant quantity when comparing the untreated model with the virtually stented model (see green circles in Figure 4). In contrast, evaluation of $R R T$ yields a more heterogeneous distribution, i.e., a smaller area with increased $R R T$ values (see asterix in Figure 4).

Regarding the underlying dynamic flow field, high OVI values predominantly occur in the fusiform environment and almost vanish after stent deployment (although not completely reduced as noticeable for the healthy case). As observed by the pathlines and due to the distal location within the Circle of Willis, the corresponding kinetic energy appears to be relatively small.

\subsection{Case 3}

Analysis of the cross-sectional area of the FIA yields a maximum area of $70.51 \mathrm{~mm}^{2}$ and a cross-sectional area of $22.61 \mathrm{~mm}^{2}$ 


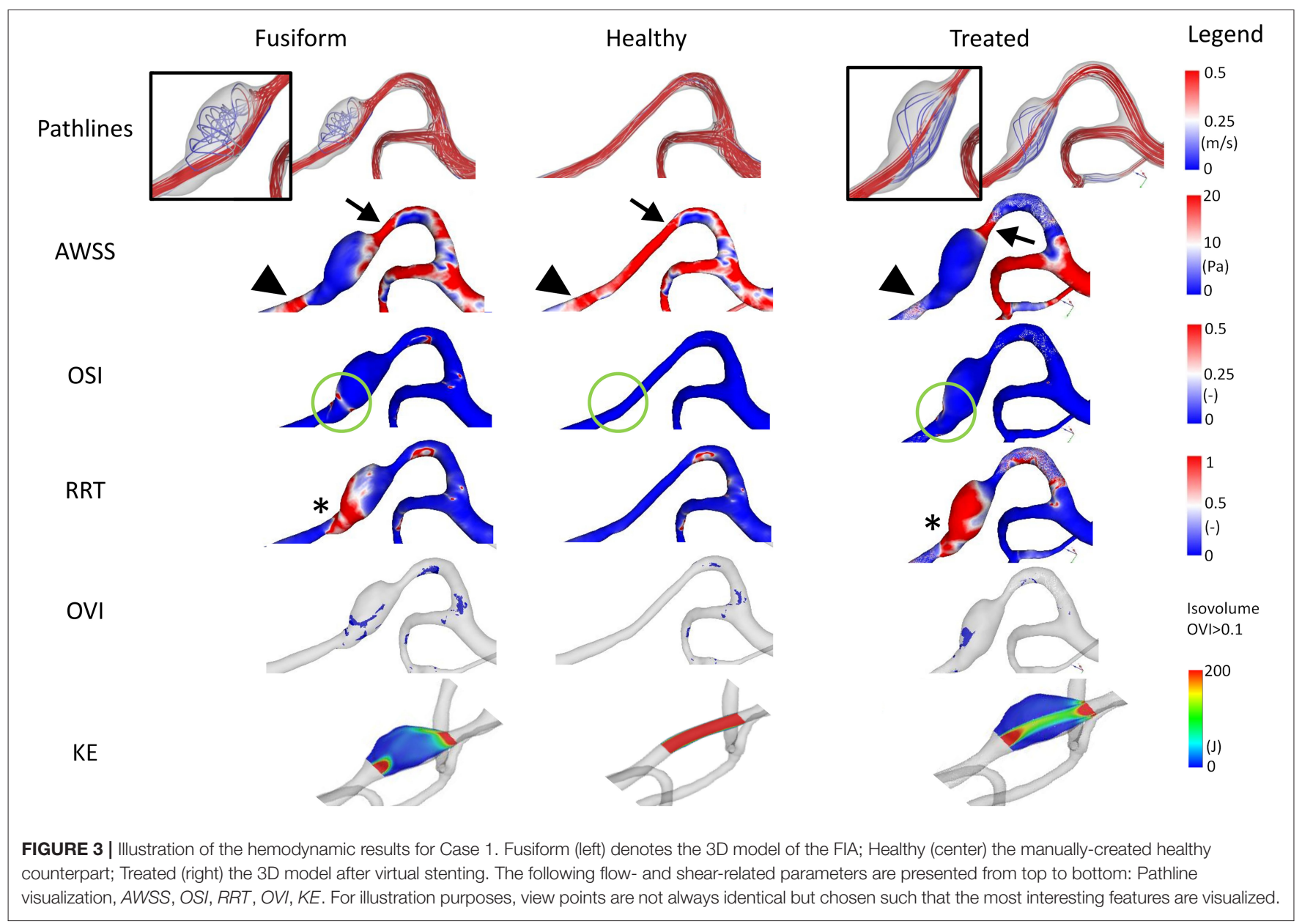

proximal to the aneurysm. Hence, dilation of the basilar artery is visible, with a relative increase of $312 \%$, recall Table 2 .

The simulated blood flow reveals high velocity values occurring in the curved basilar artery vessel section (refer to Figure 5). With the increasing growth of this region, the absolute velocity decreases, while helical flow structures develop. Regarding the AWSS analysis, a hot spot (i.e., an area with increased values) is perceivable proximal to the aneurysm (refer to arrowheads in Figure 5). Furthermore, the wall shear stress pattern occurring in the healthy-vessel section drastically changed due to the formation of the aneurysm. After virtually deploying a flow-diverting device, the low-shear stress distribution remains. However, the blood flow is directed toward the basilar tip.

The analysis of OSI reveals the existence of complex and risk-related hemodynamics for the FIA. Large patches with increased values exist which do not appear in the healthy counterpart. Impressively, the virtual treatment strongly reduces the oscillation leading to stable flow structures. For $R R T$, expected tendencies are visible. While almost no increased values appear in the healthy vessel, increased relative residence times can be noted in the fusiform and treated variant.

The flow-related analysis of $O V I$ and $K E$ confirms the observations of the previous cases. Oscillations are successfully damped by the implantation of a sufficiently-selected flowdiverting device, and the blood is desirably redirected toward the original vessel's course.

\section{DISCUSSION}

Although the research related to cerebrovascular pathologies such as intracranial aneurysms considerably increased within the last two decades, several questions related to initiation, growth, and rupture remain. Since intracranial aneurysms are mostly present in a saccular shape, fusiform cases are most typically observed when formed at the abdominal aorta. However, a small fraction of intracranial aneurysms possesses fusiform shapes as well, and depending on its appearance and localization, the identification of a sufficient (minimally-invasive) treatment strategy can be difficult.

Day et al. (21) report that hemorrhage was most common for very small lesions, whereas the incidence of bleeding is lowered with larger lesions. However, other studies reported a higher risk of hemorrhage for FIAs larger than $10 \mathrm{~mm}(22,23)$. Furthermore, a very recent MRI study by Sabotin et al. (24) demonstrated the presence of increased contrast enhancement 


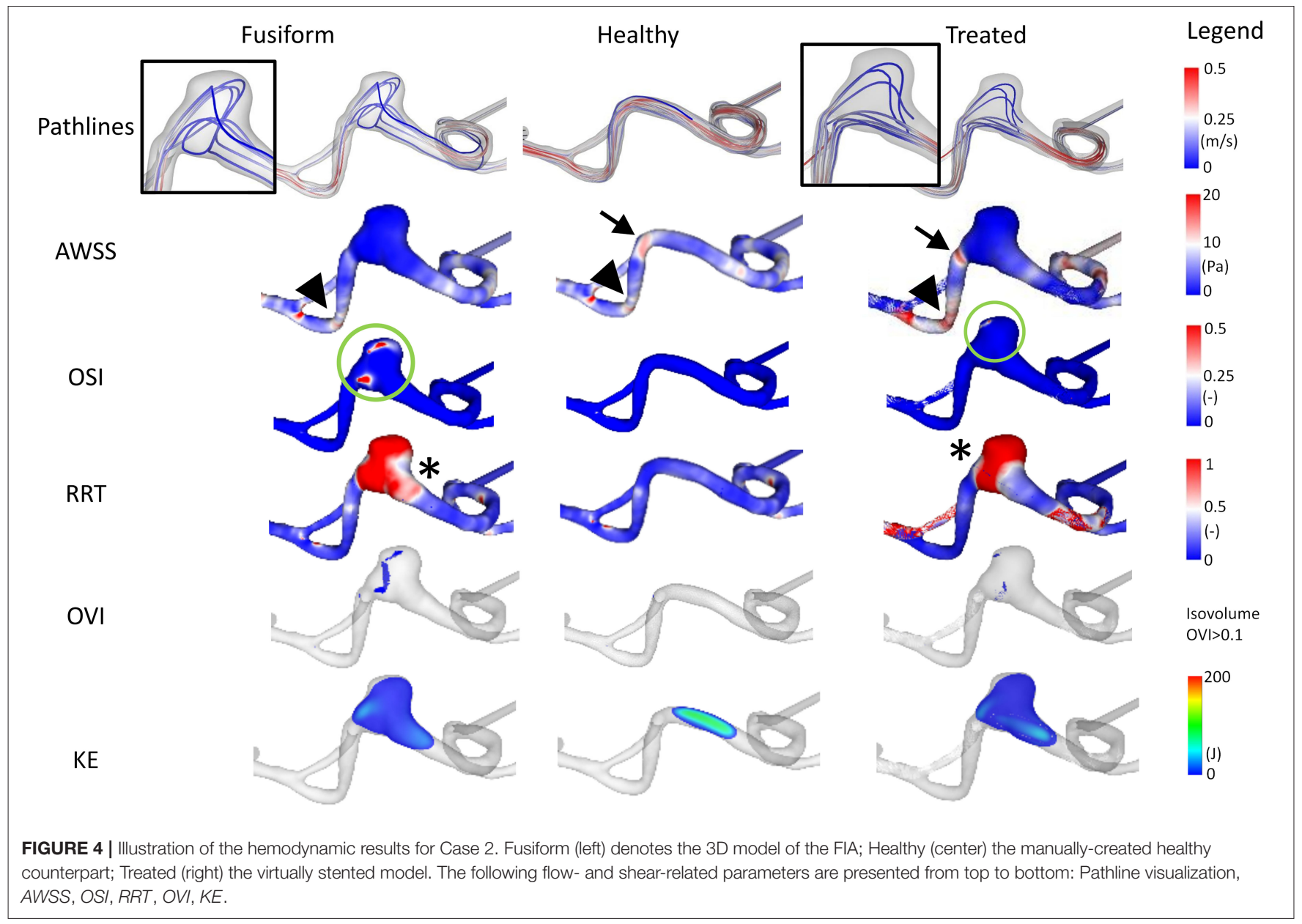

in FIAs, which was associated with microhemorrhage and pathophysiological processes.

To further improve the understanding of FIA hemodynamics, the presented pilot study focused on three representative cases due to their rare existence. The localizations are well fitting to literature, where it is stated that FIAs usually occur in the vertebrobasilar system (like Case 1 and Case 3) and that FIAs in the anterior circulation remain rare and occur mostly in the middle cerebral artery (like Case 2) (4). In addition, two of the three cases stem from men, which is also typical for FIAs.

Conducting highly-resolved hemodynamic simulations in three patient-specific FIAs enabled the assessment of locallyoccurring flow structures. Specifically, velocity-encoded pathline visualization allows for the qualitative identification of flow phenomena that might progress undesired effects, which repeatedly occur in each cardiac cycle (recall Figures 3-5). Furthermore, the precise calculation of existing AWSS patterns demonstrated that all FIAs were subject to abnormally low shear stress values, which can lead to further weakening of the vessel wall and subsequent growth or even rupture (2527). The recreation of the pre-pathological states revealed that considerably larger AWSS values were present in the regions of interest even when compared to other sections of the segmented vasculature. This observation promotes the theory that high shear is related to the initiation of intracranial aneurysms due to triggering a muralcell-mediated destructive remodeling (28).

In addition to the shear stress evaluation, the analysis of OSI, which was repeatedly associated with intracranial aneurysm rupture $(29,30)$, impressively demonstrates the effect of minimally-invasive endovascular treatment. All FIA cases were subjected to abnormally high oscillatory shear values, which were not observed in any healthy counterparts. Due to the virtual deployment of a flow-diverting device, this hemodynamically unstable environment could be restabilized leading to a strong reduction of OSI. Although RRT was correlated with aneurysm rupture at well (31), its benefit with respect to treatment outcome evaluation remains limited and this trend was not reflected in our study.

When analyzing $O V I$, i.e., a scalar field containing all temporal changes of the velocity vector directions $(20,32,33)$, the virtual treatment almost fully recovered the healthy states in all cases demonstrating the efficacy of such devices. Finally, the illustration of the kinetic energy distribution allows for the observation of the desired treatment outcome as well as the identification of undesired flow effects such as concentrated inflow jets. 


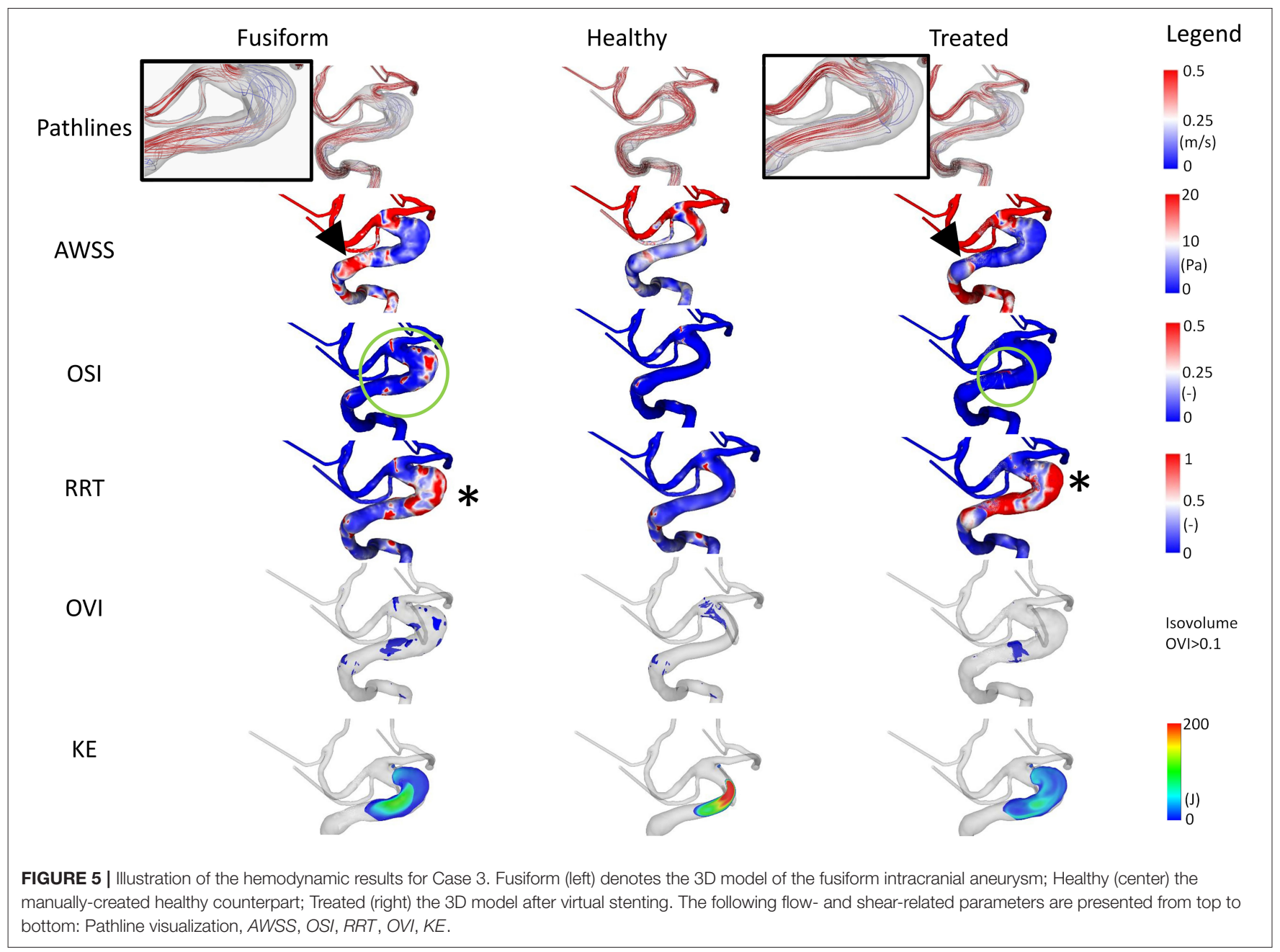

Regarding minimally-invasive therapy, stenting of FIAs is always challenging since the opening of the flow diverter differs from the behavior observable in saccular cases. Thus, the fast virtual stenting approach allows for testing different stent configurations and provides initial feedback on how strongly the stent is shortened/elongated based on the diameter along the FIA's centerline. For example, the actual stent used in Case 3 was longer than the virtually implanted flow diverter. Although other parameters such as catheter size must be taken into account, virtual planning tools provide valuable information for testing different configurations. Furthermore, undesired treatment effects such as incomplete wall apposition can be evaluated as it can be noticed for Case 1 in the distal part of the FIA (e.g., visualized by the kinetic energy distribution).

Within this study, several limitations exist. First, although our internal database comprises approximately 400 intracranial aneurysms, we were only able to identify three FIAs with sufficient image quality. Hence, only for two of them, postinterventional information was available since one patient stopped treatment. When compared to literature, the expected amount of cases would be even worse, since two large autopsy studies with a combined case number of more than 16,000 reported only 15 fusiform intracranial aneurysms $(1,2)$. Second, we conducted segmentation of $3 \mathrm{D}$ models and virtual removal of the fusiform aneurysms that include manual processing and thus might suffer from user specific variations. Third, the blood flow simulation requires several assumptions, which might not be able to perfectly reflect reality (15). However, extensive validation studies were carried out in advance to demonstrate the accuracy of image-based simulation (34). Finally, the rupture risk prediction of aneurysms is a challenging task, where longitudinal studies would be required to truly assess the individual rupture risk.

\section{CONCLUSION}

In this pilot study, we analyzed the pathological hemodynamics in three representative fusiform intracranial aneurysms based on a multi-modal simulation approach. By manually restoring the healthy hemodynamic environment for each case, reference flow conditions could be obtained for comparison. Furthermore, minimally-invasive endovascular therapy was performed in two of the patients based on a fast virtual stenting approach 
confirming the efficacy of flow-diverting devices for this neurovascular pathology.

Although we could demonstrate our findings only for a very limited number of cases due to the rarity of fusiform aneurysms, our results reflect the potential for blood flow analysis and virtual stenting of these pathologies. For future study, the analysis of a considerably larger amount of FIAs is required to confirm our initial finding.

\section{DATA AVAILABILITY STATEMENT}

The raw data supporting the conclusions of this article will be made available by the authors, without undue reservation.

\section{AUTHOR CONTRIBUTIONS}

PB and SS: conceptualization, formal analysis, resources, supervision, project administration, and funding acquisition. LM, PB, and SS: methodology. JK, JS, and SS: software. JK, LM, and $\mathrm{PB}$ : validation. JK, JS, LM, PB, and SS: investigation. OB, $\mathrm{YC}$, and $\mathrm{DB}$ : data curation. $\mathrm{PB}, \mathrm{JS}$, and SS: writing-original draft preparation and visualization. JS, LM, BP, DB, PB, and SS:

\section{REFERENCES}

1. Hayes W, Bernhardt H, Young J. Fusiform arteriosclerotic aneurysm of the basilar artery: five cases including two ruptures. Vasc Surg. (1967) 1:171-8. doi: $10.1177 / 153857446700100305$

2. Houspian E. A systemic analysis of intracranial aneurysms from the autopsy of the Prebyterian hospital. J Neuropathol Exp Neurol. (1974) 17:409-23. doi: 10.1097/00005072-195807000-00001

3. Yu Y, Moseley I, Pullicino P, McDonald W. The clinical picture of ectasia of the intracerebral arteries. J Neurol Neurosurg Psychiatry. (1982) 45:29-36. doi: 10.1136/jnnp.45.1.29

4. Park SH, Yim MB, Lee CY, Kim E, Son EI. Intracranial fusiform aneurysms: it's pathogenesis, clinical characteristics and managements. J Korean Neurosurg Soc. (2008) 44:116. doi: 10.3340/jkns.2008.44.3.116

5. Barletta EA, Ricci RL, Di Silva RG, Gaspar RHML, Araújo JFM, Neves MWF, et al. Fusiform aneurysms: a review from its pathogenesis to treatment options. Surg Neurol Int. (2018) 9:189. doi: 10.4103/sni.sni_133_18

6. Raghavan ML, Ma B, Harbaugh RE. Quantified aneurysm shape and rupture risk. J Neurosurg. (2009) 102:355-62. doi: 10.3171/jns.2005.102. 2.0355

7. Pierot L, Wakhloo AK. Endovascular treatment of intracranial aneurysms: current status. Stroke. (2013) 44:2046-54. doi: 10.1161/STROKEAHA.113.000733

8. Griessenauer CJ, Ogilvy CS, Adeeb N, Dmytriw AA, Foreman PM, Shallwani $\mathrm{H}$, et al. Pipeline embolization of posterior circulation aneurysms: a multicenter study of 131 aneurysms. J Neurosurg. (2018) 130:923-35. doi: 10.3171/2017.9.JNS171376

9. Sindeev S, Kirschke JS, Prothmann S, Frolov S, Liepsch D, Berg P, et al. Evaluation of flow changes after telescopic stenting of a giant fusiform aneurysm of the vertebrobasilar junction. Biomed Eng Online. (2019) 18:82. doi: 10.1186/s12938-019-0699-1

10. Sindeev S, Prothmann S, Frolov S, Zimmer C, Liepsch D, Berg P, et al. Intimal hyperplasia after aneurysm treatment by flow diversion. World Neurosurg. (2019) 122:e577-83. doi: 10.1016/j.wneu.2018. 10.107

11. Saalfeld S, Berg P, Niemann A, Luz M, Preim B, Beuing O. Semiautomatic neck curve reconstruction for intracranial aneurysm rupture risk assessment writing - review and editing. All the authors have read and agreed to the published version of the manuscript.

\section{FUNDING}

This study was funded by the German Federal Ministry of Education and Research within the Research Campus STIMULATE (grant no. 13GW0473A) and the German Research Foundation (grant nos. SA 3461/3-1 and BE 6230/6-1).

\section{ACKNOWLEDGMENTS}

We thank Dr. Maximilian Thormann (University hospital Magdeburg, Germany) for fruitful discussions. We acknowledge support for the Book Processing Charge by the Open Access Publication Fund of Magdeburg University.

\section{SUPPLEMENTARY MATERIAL}

The Supplementary Material for this article can be found online at: https://www.frontiersin.org/articles/10.3389/fneur. 2021.771694/full\#supplementary-material based on morphological parameters. Int J Comput Assist Radiol Surg. (2018) 13:1781-93. doi: 10.1007/s11548-018-1848-x

12. Antiga L, Piccinelli M, Botti L, Ene-Iordache B, Remuzzi A, Steinman DA. An image-based modeling framework for patient-specific computational hemodynamics. Med Biol Eng Comput. (2008) 46:1097-112. doi: 10.1007/s11517-008-0420-1

13. Janiga G, Daróczy L, Berg P, Thévenin D, Skalej M, Beuing O. An automatic CFD-based flow diverter optimization principle for patient-specific intracranial aneurysms. J Biomech. (2015) 48:3846-52. doi: 10.1016/j.jbiomech.2015.09.039

14. Berg P, Daróczy L, Janiga G. Virtual stenting for intracranial aneurysms. In: Computing and Visualization for Intravascular Imaging and ComputerAssisted Stenting. London: Elsevier (2017). p. 371-411.

15. Berg P, Saalfeld S, VoßS, Beuing O, Janiga G. A review on the reliability of hemodynamic modeling in intracranial aneurysms: why computational fluid dynamics alone cannot solve the equation. Neurosurg Focus. (2019) 47:E15. doi: 10.3171/2019.4.FOCUS19181

16. Berg P, Stucht D, Janiga G, Beuing O, Speck O, Thévenin D. Cerebral blood flow in a healthy Circle of Willis and two intracranial aneurysms: computational fluid dynamics versus four-dimensional phase-contrast magnetic resonance imaging. J Biomech Eng. (2014) 136:041003. doi: $10.1115 / 1.4026108$

17. Saalfeld S, VoßS, Preim B, Beuing O, Berg P. Flow-splitting-based computation of outlet boundary conditions for improved cerebrovascular simulation in multiple intracranial aneurysms. Int J Comput Assist Radiol Surg. (2019) 14:1805-13. doi: 10.1007/s11548-019-02036-7

18. Cebral JR, Mut F, Weir J, Putman CM. Association of hemodynamic characteristics and cerebral aneurysm rupture. Am J Neuroradiol. (2011) 32:264-70. doi: 10.3174/ajnr.A2274

19. Xiang J, Tutino VM, Snyder KV, Meng H. CFD: computational fluid dynamics or confounding factor dissemination? The role of hemodynamics in intracranial aneurysm rupture risk assessment. AJNR Am J Neuroradiol. (2014) 35:1849-57. doi: 10.3174/ajnr.A3710

20. Tanioka S, Ishida F, Kishimoto T, Tsuji M, Tanaka K, Shimosaka S, et al. Quantification of hemodynamic irregularity using oscillatory velocity index in the associations with the rupture status of cerebral aneurysms. J Neurointerv Surg. (2019) 11:614-7. doi: 10.1136/neurintsurg-2018-014489 
21. Day AL, Gaposchkin CG, Yu CJ, Rivet DJ, Dacey RG. Spontaneous fusiform middle cerebral artery aneurysms: characteristics and a proposed mechanism of formation. J Neurosurg. (2003) 99:228-40. doi: 10.3171/jns.2003.99.2.0228

22. Flemming KD, Wiebers DO, Brown RD, Link MJ, Nakatomi H, Huston J, et al. Prospective risk of hemorrhage in patients with vertebrobasilar nonsaccular intracranial aneurysm. J Neurosurg. (2004) 101:82-7. doi: $10.3171 /$ jns.2004.101.1.0082

23. Mangrum WI, Huston J, Link MJ, Wiebers DO, McClelland RL, Christianson $\mathrm{TJH}$, et al. Enlarging vertebrobasilar nonsaccular intracranial aneurysms: frequency, predictors, and clinical outcome of growth. J Neurosurg. (2005) 102:72-9. doi: 10.3171/jns.2005.102.1.0072

24. Sabotin RP, Varon A, Roa JA, Raghuram A, Ishii D, Nino M, et al. Insights into the pathogenesis of cerebral fusiform aneurysms: high-resolution MRI and computational analysis. J Neurointerv Surg. (2021) 13:1180-6. doi: 10.1136/neurintsurg-2020-017243

25. Boussel L, Rayz V, McCulloch C, Martin A, Acevedo-Bolton G, Lawton M, et al. Aneurysm growth occurs at region of low wall shear stress. Stroke. (2008) 39:2997-3002. doi: 10.1161/STROKEAHA.108.521617

26. Zhang Y, Jing L, Zhang Y, Liu J, Yang X. Low wall shear stress is associated with the rupture of intracranial aneurysm with known rupture point: case report and literature review. BMC Neurol. (2016) 16:231. doi: 10.1186/s12883-016-0759-0

27. Zhou G, Zhu Y, Yin Y, Su M, Li M. Association of wall shear stress with intracranial aneurysm rupture: systematic review and meta-analysis. Sci Rep. (2017) 7:5331. doi: 10.1038/s41598-017-05886-w

28. Meng H, Tutino VM, Xiang J, Siddiqui A. High WSS or Low WSS? Complex interactions of hemodynamics with intracranial aneurysm initiation, growth, and rupture: toward a unifying hypothesis. AJNR Am J Neuroradiol. (2014) 35:1254-62. doi: 10.3174/ajnr.A3558

29. Kawaguchi T, Nishimura S, Kanamori M, Takazawa H, Omodaka S, Sato K, et al. Distinctive flow pattern of wall shear stress and oscillatory shear index: similarity and dissimilarity in ruptured and unruptured cerebral aneurysm blebs. J Neurosurg. (2012) 117:774-80. doi: 10.3171/2012.7.JNS111991

30. Berg P, Beuing O. Multiple intracranial aneurysms: a direct hemodynamic comparison between ruptured and unruptured vessel malformations. Int J Comput Assist Radiol Surg. (2018) 13:83-93. doi: 10.1007/s11548-017-1643-0

31. Neyazi B, Swiatek VM, Skalej M, Beuing O, Stein KP, Hattingen J, et al. Rupture risk assessment for multiple intracranial aneurysms: why there is no need for dozens of clinical, morphological and hemodynamic parameters. Ther Adv Neurol Disord. (2020) 13:1756286420966159. doi: $10.1177 / 1756286420966159$

32. Bouillot P, Brina O, Ouared R, Lovblad KO, Farhat M, Pereira VM. Particle imaging velocimetry evaluation of intracranial stents in sidewall aneurysm: hemodynamic transition related to the stent design. PLoS ONE. (2014) 9:e113762. doi: 10.1371/journal.pone.0113762

33. Sano T, Ishida F, Tsuji M, Furukawa K, Shimosaka S, Suzuki H. Hemodynamic differences between ruptured and unruptured cerebral aneurysms simultaneously existing in the same location: 2 case reports and proposal of a novel parameter oscillatory velocity index. World Neurosurg. (2017) 98:868.e5-868.e10. doi: 10.1016/j.wneu.2016.12.047

34. Roloff C, Stucht D, Beuing O, Berg P. Comparison of intracranial aneurysm flow quantification techniques: standard PIV vs stereoscopic PIV vs tomographic PIV vs phase-contrast MRI vs CFD. J Neurointerv Surg. (2019) 11:275-82. doi: 10.1136/neurintsurg-2018-013921

Conflict of Interest: DB is a Proctor/Advisor: Phenox, Balt, Acandis, Thromb X, Perflow, Kaneka, Penumbra, outside of the submitted work.

The remaining authors declare that the research was conducted in the absence of any commercial or financial relationships that could be construed as a potential conflict of interest.

Publisher's Note: All claims expressed in this article are solely those of the authors and do not necessarily represent those of their affiliated organizations, or those of the publisher, the editors and the reviewers. Any product that may be evaluated in this article, or claim that may be made by its manufacturer, is not guaranteed or endorsed by the publisher.

Copyright (c) 2022 Saalfeld, Stahl, Korte, Miller Marsh, Preim, Beuing, Cherednychenko, Behme and Berg. This is an open-access article distributed under the terms of the Creative Commons Attribution License (CC BY). The use, distribution or reproduction in other forums is permitted, provided the original author(s) and the copyright owner(s) are credited and that the original publication in this journal is cited, in accordance with accepted academic practice. No use, distribution or reproduction is permitted which does not comply with these terms. 